\title{
Validation des données périnatales de la Base de données sur les congés des patients de l'Institut canadien d'information sur la santé
}

\author{
K.S. Joseph, M.D., Ph.D. (1) et J. Fahey, M.Math (2) pour le Système canadien de surveillance périnatale
}

\section{Résumé}

Nous avons comparé les données périnatales consignées dans la base de données sur les hospitalisations de l'Institut canadien d'information sur la santé (ICIS) à celles de la Nova Scotia Atlee Perinatal Database (NSAPD) afin d'évaluer l'exactitude des renseignements de l'ICIS. Le codage des interventions, comme les césariennes, est exact (sensibilité de 99,8 \% et spécificité de 98,7\%). L’hémorragie du post-partum, le déclenchement artificiel du travail et l'hémorragie intraventriculaire grave avaient également des taux de sensibilité et de spécificité de plus de $85 \%$ et de plus de $95 \%$, respectivement. Le codage de certains diagnostics dont les définitions diffèrent dans les deux bases de données était moins exact. Citons le cas du syndrome de détresse respiratoire (SDR) qui avait une sensibilité de 50,9\% et une spécificité de 99,8\%. L'exactitude du codage peut être améliorée en limitant l'analyse aux formes les plus graves de la maladie. Par exemple, on obtient une sensibilité de $100 \%$ et une spécificité de 99,6 \% en limitant le SDR au SDR grave dans la NSAPD et en combinant les codes du SDR et de l'intubation afin d'identifier le SDR grave dans la base de données de l'ICIS. Notre étude cautionne l'utilisation des données de l'ICIS pour la surveillance nationale de la morbidité périnatale, à condition que leur interprétation soit facilitée par une compréhension de la pratique clinique et par des analyses de sensibilité visant à assurer la fiabilité des résultats.

Mots clés : périnatalité, surveillance, base de données, maternité, nouveau-né, morbidité, détresse respiratoire, base de données sur les congés des patients

\section{Introduction}

Au Canada, la surveillance de la santé périnatale repose sur des données tirées de diverses sources, notamment des bases de données sur l'état civil et des bases de données sur les congés d'hôpital ${ }^{1-3}$. Même si les statistiques de l'état civil sur les naissances, les décès de nourrissons et la mortalité intra-utérine sont encore une source importante de renseignements à propos des tendances temporelles et des variations régionales relatives à la santé périnatale, la surveillance périnatale est de plus en plus axée sur les tendances et la répartition des cas de morbidité grave en raison de la diminution du taux de mortalité des dernières décennies. Cette constatation s'applique particulièrement aux cas de morbidité maternelle grave ${ }^{4}$ et de morbidité néonatale grave ${ }^{5,6}$.

La qualité des données de la Base de données sur les congés des patients (BDCP) de l'Institut canadien d'information sur la santé (ICIS) est une préoccupation centrale. Même si des études antérieures ont montré que ces données conviennent à la surveillance ${ }^{7}$, une étude récente de réextraction des données des dossiers médicaux, commandée par le Système canadien de surveillance périnatale (SCSP) et exécutée par l'ICIS, a montré que la qualité des données varie pour plusieurs indicateurs de la santé périnatale ${ }^{8}$. L'étude sur les congés d'hôpital de 19992000 portait sur des hôpitaux choisis aléatoirement dans des strates définies en fonction de la situation géographique et de la taille et du type d'établissement. Les données des dossiers de 385 nouveau-nés et de 872 mères ont été extraites de nouveau par des spécialistes de la classification de l'ICIS, puis comparées aux données de la BDCP. Le taux de faux positifs était inférieur à $1 \%$ pour la césarienne, l'accouchement avec forceps ou ventouse, l'accouchement prématuré et l'épisiotomie. Toutefois, le taux de faux positifs d'autres indicateurs était élevé (p. ex. 12,8 \% pour le déclenchement artificiel du travail et $40,3 \%$ pour la déchirure périnéale du troisième degré). Le taux de faux négatifs était également faible pour certains indicateurs (p. ex. il était inférieur à $1 \%$ pour la césarienne, la déchirure du troisième degré, le syndrome de détresse respiratoire $[\mathrm{SDR}]$, la prééclampsie et l'éclampsie), mais élevé pour d'autres indicateurs (p. ex. 23,6\% pour l'asphyxie ou la détresse fœtale; 38,3 \% pour le déclenchement artificiel du travail; $41,3 \%$ pour les pathologies néonatales rares et 53,9 \% pour les pathologies congénitales rares).

Coordonnées des auteurs

1 Unité de recherche en épidémiologie périnatale, Département d’obstétrique, de gynécologie et de pédiatrie, Université Dalhousie et Centre de soins de santé IWK, Halifax (Nouvelle-Écosse)

2 Reproductive Care Program of Nova Scotia (programme de soins en matière de reproduction de la Nouvelle-Écosse), Halifax (Nouvelle-Écosse)

Correspondance : K.S. Joseph, M.D., Ph.D., Division of Neonatal-Perinatal Medicine, IWK Health Centre, 5980 University Avenue, Halifax (Nouvelle-Écosse) B3K 6R8, Tél. : 902-470-6652; Téléc. : 902-470-7190; Courriel : ksjoseph@dal.ca 
Deux préoccupations ont été exprimées par le Groupe d'étude sur la santé fœtale et infantile et le Groupe d'étude sur la santé maternelle du Système canadien de surveillance périnatale (SCSP) qui utilisent régulièrement les données de l'ICIS à des fins de surveillance ${ }^{9-13}$. D'abord, l'analyse détaillée de phénomènes tels que l'embolie amniotique ${ }^{12}$, l'hémorragie du post-partum $^{13}$ et d'autres conditions ${ }^{9-11}$ montre des tendances qui correspondent à ce qu'on s'attend à voir en clinique, ce qui porte à croire que les données sont d'une qualité supérieure à celle qui a été observée dans le cadre de l'étude de réextraction. Une autre raison de remettre en question le taux d'erreurs élevé noté dans l'étude de réextraction concerne les aspects techniques de l'échantillonnage stratifié (et au calcul pondéré des taux de faux positifs et de faux négatifs dans la population). Plusieurs taux d'erreurs étaient relativement faibles pour l'échantillon, mais étaient considérablement gonflés après la pondération. Les augmentations les plus importantes ont été observées pour la déchirure périnéale du troisième degré (taux de faux positif de 5,6\% dans l'échantillon et de $40,3 \%$ dans la population), pour les pathologies néonatales rares (taux de faux négatifs de 1,6\% dans l'échantillon et de $41,3 \%$ dans la population) et pour les pathologies congénitales rares (taux de faux négatifs de 3,1\% dans l'échantillon et de 53,9\% dans la population).

Par conséquent, nous avons décidé de réévaluer l'exactitude des renseignements de la BDCP en comparant les données périnatales de cette base avec celles de la NSAPD. Le degré d'exactitude de cette dernière, qui est plus petite et axée sur la clinique, est considéré comme relativement élevé en ce qui à trait aux diagnostics et aux interventions.

\section{Méthode}

Pendant une courte période en 2002, les données périnatales concernant les nouveaunés et les mères en Nouvelle-Écosse ont été simultanément codées dans la BDCP et la NSAPD. Malgré les différences entre les règles de codage de ces bases de données, l'accessibilité des données sur 6194 mères et 6315 nouveau-nés dans deux systèmes indépendants a permis d'évaluer la qualité des données de la BDCP. Ces mères et ces nouveau-nés n’ont pas été sélectionnés en fonction de l'âge gestationnel ou du déroulement et de l'issue de la grossesse, et représentent tous les accouchements/ naissances qui ont eu lieu au cours d'une période déterminée.

La NSAPD est une base de données populationnelle axée sur la clinique qui renferme des renseignements détaillés tirés des dossiers prénatals et médicaux. Ces renseignements sont extraits par du personnel qualifié à l'aide de formulaires normalisés. Un programme continu de contrôle de la qualité des données, qui comprend des études d'extraction périodiques, a confirmé la fiabilité des renseignements de la base de données. Cette base a servi à valider les statistiques de l'état civil, et plus particulièrement le fichier de données couplées naissance-décès de Statistique Canada $^{14,15}$. Les données périnatales de la BDCP ont également été recueillies en Nouvelle-Écosse par du personnel qualifié, qui a suivi les règles d'extraction des données de l'ICIS. Au cours de cette période, les renseignements diagnostiques ont été codés selon la Classification internationale des maladies, $10^{\mathrm{e}}$ révision (CIM-10-CA) et les renseignements sur les interventions, selon la Classification canadienne des interventions en santé (CCI).

Nous avons comparé les diagnostics et les interventions pertinents dans les deux bases de données, en supposant que la NSAPD constitue la référence. Le taux de sensibilité (c.-à-d. la proportion de vrais positifs [NSADP] également déterminés comme positifs dans la BDCP) et le taux de spécificité (c.-à-d. la proportion de vrais négatifs [NSADP] également déterminés comme négatifs dans la BDCP) ont été calculés pour des intervalles de confiance (IC) binominaux exacts à $95 \%$. L'estimation de l'âge gestationnel consigné dans les deux sources a également été évaluée en utilisant la concordance statistique (c.-à-d. le kappa pondéré et le coefficient de corrélation interclasse). Des groupes d'âge gestationnel ont été établis selon les catégories pronostiques pertinentes en pratique clinique couramment utilisées par le Système canadien de surveillance périnatale ( $<20,20$ à 21,22 à 23,24 à 25, 26 à 27, 28 à 31,32 à 33,34 à 36, 37 à 41,42 à 45 semaines et non disponible). Les diagnostics et les interventions utilisés pour l'évaluation des données de la BDCP ont été sélectionnés en fonction de leur pertinence en clinique et en santé publique, et de la compatibilité de leurs définitions dans les deux bases de données.

\section{Résultats}

Selon la BDCP, le taux d'accouchements prématurés (c.-à-d. la proportion de femmes chez qui on connaît l'âge gestationnel et qui accouchent avant la fin de la $37^{\mathrm{e}}$ semaine de grossesse) était de 9,1\% (IC à $95 \%$ : de 8,4 à 9,9\%), alors que selon la NSAPD ce taux était de 8,8 \% (IC à $95 \%$ : de 8,1 à 9,6 \%). Le taux d'accouchements après terme (c.-à-d. d'accouchements à 42 semaines complètes ou plus de grossesse) était de 0,6\% (IC à $95 \%$ : de 0,5 à $0,9 \%$ ) et de $2,1 \%$ (IC à $95 \%$ : de 1,8 à 2,5 \%) selon la BDCP et la NSAPD, respectivement. L'âge gestationnel n'était pas connu pour 54 femmes selon la NSAPD (l'âge gestationnel de 47 de ces femmes se situait entre 37 et 41 semaines selon la BDCP) et pour 7 femmes selon la BDCP. Selon la BDCP, 495 femmes ont accouché prématurément, comparativement aux 543 cas recensés dans la NSAPD (ce qui correspond à une sensibilité de 91,2 \%). Pour les accouchements à terme ou après terme, on trouve 5531 cas dans la BDCP, par rapport à 5597 dans la NSAPD (spécificité de 98,8\%). Une analyse détaillée des données sur les accouchements prématurés montre que 64 des 66 (97\%) femmes à qui on avait attribué un code d'accouchement prématuré dans la BDCP (mais un code d'accouchement à terme ou après terme dans la NSAPD) avaient un âge gestationnel de 36 semaines. Une grande proportion des faux positifs était donc causée par une différence mineure, soit une semaine, dans l'âge gestationnel. De plus, 31 des 48 (65\%) femmes qui ont accouché prématurément selon la NSAPD, mais à terme ou après terme selon la BDCP, avaient un âge gestationnel de 37 semaines selon la BDCP. Le kappa pondéré évaluant la concordance des données sur l'âge gestationnel de la BDCP et de la NSAPD est de 0,75 (IC à $95 \%$ : de 0,72 à 0,78 ) et 
TABLEAU 1

Validité des données sur la santé maternelle tirées de la Base de données sur les congés des patients de l'Institut canadien d'information sur la santé, en fonction des données de la Nova Scotia Atlee Perinatal Database (données de référence, basées sur 6194 mères; Nouvelle-Écosse, 2002)

\begin{tabular}{|c|c|c|c|c|}
\hline Indicateur & Sensibilité (\%) & IC à $95 \%$ & Spécificité (\%) & IC à $95 \%$ \\
\hline Accouchement prématuré (avant 37 semaines) & 91,2 & $88,5-93,4$ & 98,8 & $98,5-9,1$ \\
\hline Hémorragie du post-partum & 90,2 & $86,2-93,3$ & 98,2 & $97,8-98,5$ \\
\hline Déclenchement du travail & 89,2 & $87,7-90,6$ & 96,9 & $96,4-97,4$ \\
\hline Césarienne & 99,8 & $99,5-100,0$ & 98,7 & $98,3-99,0$ \\
\hline$-3^{\mathrm{e}}$ degré & 97,1 & $92,7-99,2$ & 99,9 & $99,8-100,0$ \\
\hline$-4^{e}$ degré & 94,7 & $74,0-99,7$ & 99,9 & $99,8-100,0$ \\
\hline Hypertension chronique & 83,3 & $73,6-90,6$ & 99,9 & $99,8-100,0$ \\
\hline $\begin{array}{l}\text { Hypertension gravidique avec protéinurie } \\
\text { (p. ex. HG ou HEHRP) }\end{array}$ & 75,2 & $67,5-81,8$ & 99,5 & $99,3-99,7$ \\
\hline
\end{tabular}

Un accouchement prématuré a lieu avant la fin de la $37^{\text {e }}$ semaine de grossesse.

HG : hypertension gravidique

HEHRP : hémolyse, enzymes hépatiques élevées et nombre réduit de plaquettes

le cœefficient de corrélation interclasse, de 0,86 (IC à $95 \%$ : de 0,83 à 0,88 ).

Le tableau 1 présente les taux de sensibilité et de spécificité de plusieurs indicateurs de la santé maternelle. Le degré d'exactitude de la plupart des indicateurs de la BDCP est élevé. Des taux de sensibilité de $85 \%$ à $90 \%$ ont été notés pour les transfusions sanguines, le déclenchement du travail et tous les types d'hypertension gravidique. Le codage de bon nombre d'interventions et d'agents de déclenchement du travail dans la BDCP concordait avec le codage du déclenchement du travail dans la NSAPD. Par exemple, les 29 femmes dont le travail a été déclenché selon le codage de la BDCP, par divers moyens, notamment un agent ocytocique, ont également reçu le code de déclenchement du travail dans la NSAPD. La principale divergence entre les deux bases de données concernait des cas où le code de la BDCP correspondait au déclenchement du travail par rupture artificielle des membranes, tandis que plusieurs de ces cas (45 sur 191) se sont vu attribuer par la NSAPD un code désignant une rupture artificielle des membranes, mais sans déclenchement du travail. Étant donné que la rupture artificielle des membranes après le début du travail ne correspond pas à un déclenchement du travail, cette divergence est probablement le fruit d'une erreur de codage dans la BDCP (si on présume que l'information de la NSAPD est exacte). Des écarts ont également été notés en ce qui concerne le diagnostic d'hypertension gravidique. Nous nous y attendions en raison des divers types d'hypertension gravidique et des systèmes de codage et de classification différents de la NSAPD et de la CIM-10-CA.

Le tableau 2 présente l'évaluation des indicateurs de la santé des nouveau-nés chez les 6315 nourrissons. Le taux de faux négatifs pour la septicémie bactérienne était élevé (sensibilité de 38,4\%). Ce taux a diminué lorsque nous avons cherché, dans la BDCP, les codes désignant la septicémie chez les adultes en plus des codes correspondant à la septicémie chez les nouveau-nés. Les stades les plus graves d'hémorragie intraventriculaire et les fractures de la clavicule étaient correctement codés dans la BDCP, mais le codage du SDR montrait des divergences semblables à celles du codage de l'hypertension gravidique (en effet, la classification du SDR est très détaillée dans la NSAPD et diffère de celle de la CIM-10-CA). Néanmoins, en combinant le code du SDR de la CIM-10CA et le code d'intubation de la CCI dans la BDCP, on obtenait une correspondance presque parfaite avec le diagnostic de SDR grave de la NSAPD. En effet, dans la NSAPD, le code du SDR grave correspond à un SDR nécessitant une ventilation assistée. L'asphyxie fœtale ou à la naissance ne figure pratiquement pas dans la BDCP (sensibilité de $14,3 \%$, voir le tableau 2).

\section{Analyse}

Notre étude a confirmé que le codage des interventions majeures, par exemple de la césarienne, était valide dans la BDCP de l'ICIS. Elle a également montré que les données sur les diagnostics mineurs (p. ex. les déchirures périnéales du premier au quatrième degré) et sur les diagnostics et les interventions plus complexes (p. ex. le déclenchement du travail, qui est facilement confondu avec une accélération du travail) étaient également raisonnablement exactes. De plus, les données relatives à l'âge gestationnel - mesure difficile à cerner avec exactitude en raison de la diversité des méthodes utilisées - exhibent un assez fort degré de concordance entre les deux bases. Le taux général de naissances prématurées n'était pas significativement plus élevé 
TABLEAU 2

Validité des données sur la santé des nouveau-nés tirées de la Base de données sur les congés des patients de l'Institut canadien d'information sur la santé, en fonction des données de la Nova Scotia Atlee Perinatal Database

(données de référence, basées sur 6315 naissances vivantes; Nouvelle-Écosse, 2002)

\begin{tabular}{|c|c|c|c|c|}
\hline Indicateur & Sensibilité (\%) & IC à $95 \%$ & Spécificité (\%) & IC à $95 \%$ \\
\hline Septicémie bactérienne & 38,4 & $28,1-49,5$ & 99,7 & $99,5-99,8$ \\
\hline $\begin{array}{l}\text { Septicémie bactérienne (codes pour les adultes et } \\
\text { les nouveau-nés) }\end{array}$ & 67,4 & $56,5-77,2$ & 99,6 & $99,4-99,8$ \\
\hline Hémorragie intraventriculaire, stades 3 et 4 & 88,9 & $51,8-99,7$ & 100,0 & $99,9-100,0$ \\
\hline Fracture de la clavicule & 91,7 & $61,5-99,8$ & 100,0 & $99,3-100,0$ \\
\hline - SDR (vs SDR) & 50,9 & $43,1-58,6$ & 99,8 & $99,7-99,9$ \\
\hline - SDR (vs SDR grave) & 96,3 & $89,6-99,2$ & 99,6 & $99,4-99,8$ \\
\hline $\begin{array}{l}\text { - SDR, n'importe quel } \\
\text { code + intubation } \\
\text { (vs code du SDR grave) }\end{array}$ & 100,0 & $95,5-100,0$ & 99,6 & $99,4-99,8$ \\
\hline
\end{tabular}

N'importe quel code vs n'importe quel code correspond à l'évaluation de l'un ou l'autre des codes de détresse respiratoire de l'ICIS en fonction de l'un ou l'autre des codes de détresse respiratoire de la NSAPD.

SDR vs SDR correspond à l'évaluation du code du syndrome de détresse respiratoire de l'ICIS en fonction du code du syndrome de détresse respiratoire de la NSAPD.

SDR vs SDR grave correspond à l'évaluation du code du syndrome de détresse respiratoire de l'ICIS en fonction du code du syndrome de détresse respiratoire grave de la NSAPD.

SDR, tous les codes + intubation correspond à l'évaluation des codes de syndrome de détresse respiratoire et d'intubation de l'ICIS en fonction du code du syndrome respiratoire grave de la NSAPD.

dans la BDCP que dans la NSAPD, alors que le taux de naissances après terme était significativement plus faible. Même si ces différences sont relativement mineures, leur nature suggère une utilisation plus importante de l'échographie en début de grossesse pour estimer l'âge gestationnel (et, par conséquent, une plus grande précision) dans la BDCP. Comparativement à l'estimation de l'âge gestationnel basée sur la date des dernières règles, l'estimation par échographie précoce tend à faire augmenter légèrement le taux de naissances prématurées et à faire diminuer considérablement le nombre de naissances après terme ${ }^{16-18}$. Les divergences entre les deux bases de données étaient en général liées à des diagnostics dont la définition varie, par exemple la septicémie bactérienne et le SDR. Néanmoins, on a pu obtenir des données relativement exactes dans la BDCP en utilisant aussi bien les codes pour les adultes que les codes pour les nouveau-nés dans le cas de la septicémie bactérienne et en limitant le SDR à «tous les problèmes respiratoires » ou à une forme grave du SDR.

La divergence importante entre les données de la NSAPD et de la BDCP concernant l'asphyxie fœtale ou à la naissance était prévue et ne reflète pas une inexactitude des données de la BDCP. Des études ont montré que ces termes ont presque disparu ${ }^{19}$ $\mathrm{du}$ langage clinique en raison du risque de poursuites pour faute professionnelle, même si la fréquence de cette pathologie est pratiquement inchangée ${ }^{20}$. En conséquence, ce code est rarement inscrit dans la BDCP, où les encodeurs l'utilisent seulement si le terme " asphyxie » est inscrit au dossier médical. Par contre, dans la NSAPD, cette pathologie est encore codée selon ses manifestations cliniques.

Cette étude a été menée à partir des dossiers médicaux de 2002, année où la NouvelleÉcosse a commencé l'implantation des codes de la CIM-10-CA dans les hôpitaux. Étant donné que la CIM-10-CA est substantiellement différente de la version précédente, certaines erreurs de codage ayant eu lieu en 2002 auraient été corrigées après la période de rodage du système. Par exemple, le codage de la septicémie bactérienne chez les nouveau-nés affichait une faible sensibilité en partie parce que les codes correspondant à la septicémie chez l'adulte ont aussi été utilisés. Une plus grande familiarité avec le système de codage de la CIM-10-CA aurait vraisemblablement permis d'éviter ces erreurs.

L'amélioration de l'exactitude liée au diagnostic des maladies, telle que celle obtenue en limitant le SDR aux formes les plus graves de la maladie dans la BDCP, porte à croire que les chercheurs utilisant les bases de données volumineuses sur les hospitalisations devraient régulièrement réaliser des analyses de sensibilité pour évaluer la robustesse de leurs résultats. Une bonne connaissance de la culture clinique et des changements des habitudes des cliniciens (notamment savoir quels termes sont moins utilisés, comme dans le cas d'" asphyxie à la naissance ») ${ }^{19,20}$ est un facteur important pour interpréter correctement les tendances dégagées de ces bases de données. La recherche multidisciplinaire est probablement l'approche la plus appropriée.

Une limite de notre étude tient au fait que, dans la plupart des cas en Nouvelle-Écosse, les mêmes employés ont été chargés du codage des dossiers médicaux dans la BDCP et dans la NSAPD. La concordance 
des deux bases est donc peut-être plus importante que si les deux systèmes étaient totalement indépendants. Par contre, des règles de codage détaillées existent pour chaque système (y compris l'application de critères relatifs au diagnostic et aux sections spécifiques du dossier médical d'où les données doivent être extraites). L'effet de ces règles de codage ne fait pas de doute si l'on considère les divergences de diagnostic dans les cas où aucun critère normalisé n'est appliqué.

Une autre limite potentielle de notre étude découle de l'hypothèse selon laquelle les données de la NSAPD, une base plus petite, axée sur la clinique et dotée d'un programme de contrôle de la qualité, sont plus exactes que celles de la BDCP. Il est peu probable que cette hypothèse, même si elle est défendable, soit valide pour tous les renseignements contenus dans les deux bases. Par exemple, les données sur le sang et les produits sanguins recueillies dans la base de données de l'ICIS sont plus détaillées que celles de la NSAPD. De plus, l'algorithme utilisé dans la NSAPD pour déterminer l'âge gestationnel s'appuie seulement sur la date des dernières menstruations et l'examen pédiatrique du nouveau-né. L'âge gestationnel inscrit dans la BDCP pourrait représenter une meilleure estimation étant donné qu'il tient compte des renseignements obtenus par une échographie précoce ${ }^{16-18}$.

Finalement, notre étude était limitée à une seule province canadienne. Ses résultats ne sont peut-être donc pas généralisables aux autres provinces ou territoires, ou même à un autre moment en Nouvelle-Écosse. Néanmoins, le degré relativement élevé d'exactitude observé dans notre étude est encourageant et plus conforme aux attentes des enquêteurs du SCSP qui ont travaillé à partir des données de la $\mathrm{BDCP}^{9-13}$ que les résultats de l'étude de réextraction ${ }^{8}$.

En résumé, notre étude comparait les données de la BDCP avec celles d'une base de données plus petite et axée sur la clinique. Elle a montré que les données de la BDCP étaient exactes pour un grand nombre de diagnostics et d'interventions analysés. De plus, les données moins exactes sur certains diagnostics, généralement les pathologies dont la définition fluctue, peuvent être améliorées en utilisant une combinaison de codes et en limitant la maladie à ses formes les plus graves. Cette étude cautionne donc le recours aux données de la BDCP de l'ICIS pour la surveillance et la recherche périnatales à l'échelle nationale, à condition que l'on se fonde, dans l'interprétation des données sur la compréhension de la pratique clinique et que la robustesse des résultats soit validée par des analyses de sensibilité.

\section{Remerciements}

Cette étude a été réalisée grâce à la contribution financière de la Section de la santé maternelle et infantile, Division de la surveillance de la santé et de l'épidémiologie de l'Agence de la santé publique du Canada. Nous remercions les responsables du Reproductive Care Program (programme de soins en matière de reproduction) de la Nouvelle-Écosse de nous avoir fourni l'accès à leurs données.

\section{Références}

1. Santé Canada. Rapport sur la santé périnatale au Canada, Ottawa, Travaux publics et Services gouvernementaux Canada, 2000.

2. Santé Canada. Rapport sur la santé périnatale au Canada, Ottawa, Travaux publics et Services gouvernementaux Canada, 2003.

3. Agence de la santé publique du Canada. Rapport sur la santé périnatale au Canada, éd. 2008, Ottawa, Travaux publics et Services gouvernementaux Canada, 2008.

4. Wen SW, Huang L, Liston R, et al. Severe maternal morbidity in Canada, 1991-2001. CMAJ. 2005;173(7)759-64.

5. Wilson-Costello D, Friedman H, Minich N, Fanaroff AA, Hack M. Improved survival rates with increased neurodevelopmental disability for extremely low birth weight infants in the 1990s. Pediatrics. 2005; 115:997-1003.
6. Wilson-Costello D, Friedman H, Minich N, Siner B, Taylor G, Schluchter M, Hack M. Improved neurodevelopmental outcomes for extremely low birth weight infants in 2000- 2002. Pediatrics. 2007;119:37-45.

7. Wen SW, Liu S, Marcoux S, Fowler D. Uses and limitations of routine hospital admission/separation records for perinatal surveillance. Chronic Dis Can. 1997; 18:113-9.

8. Santé Canada. An evaluation of the quality of obstetric/neonatal discharge abstract data by reabstraction of medical charts, Ottawa, Santé Canada, 2003.

9. Liu S, Heaman M, Kramer MS, Demissie K, Wen SW, Marcoux S. Length of hospital stay, obstetric conditions at childbirth, and maternal readmission: a populationbased cohort study. Am J Obstet Gynecol. 2002;187:681-7.

10. Liu S, Heaman M, Joseph KS, et al. Risk of maternal postpartum readmission associated with mode of delivery. Obstet Gynecol. 2005;105:836-42.

11. Liu S, Liston RM, Joseph KS, Heaman M, Sauve R, Kramer MS. Maternal mortality and severe morbidity associated with low-risk planned cesarean delivery versus planned vaginal delivery at term. CMAJ. 2007;176:455-60.

12. Kramer MS, Rouleau J, Baskett TF, Joseph KS. Amniotic-fluid embolism and medical induction of labour: a retrospective, population-based cohort study. Lancet. 2006;368:1444-8.

13. Joseph KS, Rouleau J, Kramer MS, Young DC, Liston RM, Baskett TF. Investigation of an increase in postpartum haemorrhage in Canada. BJOG. 2007;114:751-9.

14. Fair M, Cyr M, Allen AC, Wen SW, Guyon G, MacDonald RC. An assessment of the validity of a computer system for probabilistic record linkage of birth and infant death records in Canada. Chronic Dis Can. 2000;21:8-13. 
15. Fair M, Cyr M. Allen AC, Wen SW, Guyon G, Macdonald RC. Validation study for a record linkage of births and infant deaths in Canada, Ottawa, Statistique Canada, 1999.

16. Kramer MS, McLean FH, Boyd ME, Usher RH. The validity of gestational age estimation by menstrual dating in term, preterm, and postterm gestations. JAMA. 1988;260:3306-8.

17. Goldenberg RL, Davis RO, Cutter GR, Hoffman HJ, Brumfield CG, Foster JM. Prematurity, postdates, and growth retardation: the influence of use of ultrasonography on reported gestational age. Am J Obstet Gynecol. 1989;160:462-70.

18. Joseph KS, Huang L, Liu S, Ananth CV, Allen AC, Sauve R, Kramer MS. Reconciling the high rates of preterm and postterm birth in the United States. Obstet Gynecol. 2007;109:813-22.

19. Wu YW, Backstrand KH, Zhao S, Fullerton HJ, Johnston SC. Declining diagnosis of birth asphyxia in California: 1991-2000. Pediatrics. 2004;114:1584-90.

20. Dzakpasu S, Joseph KS, Huang L, Allen A, Sauve R, Young D. Decline in birth asphyxia in Canada: fact or artefact. Pediatrics. 2009;123:e668-72. 